\title{
A Wide Adsorption Range Hybrid Material Based on Chitosan, Activated Carbon and Montmorillonite for Water Treatment
}

\author{
Farida Bouyahmed ${ }^{1}$, Min Cai ${ }^{1}$, Laurence Reinert ${ }^{2}$, Laurent Duclaux ${ }^{2}$, Ratan Kumar Dey ${ }^{3}$, \\ Hicham Ben Youcef ${ }^{4}$, Mohammed Lahcini ${ }^{5}$, Fabrice Muller ${ }^{6}$ and \\ Sandrine Delpeux-Ouldriane ${ }^{1, *}$ \\ 1 CNRS-ICMN, Université d'Orléans, 45071 Orléans, France; farida.bouyahmed@cnrs-orleans.fr (F.B.); \\ min.cai@cnrs-orleans.fr (M.C.) \\ 2 LCME, Université Savoie Mont Blanc, 73000 Chambéry, France; laurence.reinert@univ-savoie.fr (L.R.); \\ laurent.duclaux@univ-smb.fr (L.D.) \\ 3 Centre for Applied Chemistry/Centre for Nanotechnology, Central University of Jharkhand, Brambe, \\ Jharkhand, 835205 Ranchi, India; rkdey@rediffmail.com \\ 4 Materials Science and Nano-engineering Department, Mohammed VI Polytechnic University, \\ 43150 Ben Guerir, Morocco; Hicham.BENYOUCEF@um6p.ma \\ 5 Laboratory of Organometallic and Macromolecular Chemistry-Composites Materials, Faculty of Sciences \\ and Technologies of Marrakech, CADAI AYYAD University, 40000 Marrakech, Morocco; \\ m.lahcini@uca.ac.ma \\ 6 ISTO, CNRS-Université d'Orléans-BRGM, 45071 Orléans, France; fabrice.muller@univ-orleans.fr \\ * Correspondence: delpeux@cnrs-orleans.fr; Tel.: +33-238257872
}

Received: 21 March 2018; Accepted: 17 May 2018; Published: 5 June 2018

\begin{abstract}
Numerous adsorbent materials are developed and are able to face specific types of pollution, but none of them can manage the whole pollution. The purpose of this work is to develop a novel hybrid adsorbent, based on chitosan (CS) biopolymer, clay minerals and activated carbon (AC), having complementary adsorption properties and achieving a wide-spectrum water decontamination in a single treatment. Hybrid CS beads, containing dispersed clay and AC, were prepared from dispersions of solid adsorbents in a CS solution and its further coagulation in a basic medium. The porosity and the homogeneity of the hybrid beads were characterized by $\mathrm{N}_{2}$ adsorption at $77 \mathrm{~K}$ and Cryo-Scanning Electron Microscopy respectively. The interaction between CS and clay was characterized using X-ray diffraction. Water content and the amount of each adsorbent in the hydrogel beads were determined by thermogravimetric analysis. Such a composite material was still porous and presented a wide adsorption spectrum. As shown by their adsorption kinetics, hydrophobic anionic clofibric acid (CBA) and cationic metoprolol (MTP) were well adsorbed on AC containing beads ( 21 and $26 \mathrm{mg} / \mathrm{g}$ ), respectively. Clays containing beads showed interesting adsorption properties towards cationic $\mathrm{Zn}^{2+}$ and MTP. The obtained composite beads were able to adsorb all the pollutant types: Zinc cations, and hydrophobic-charged organic molecules, such as pharmaceutical derivatives (clofibric acid and MTP).
\end{abstract}

Keywords: adsorption; biopolymer; clay minerals; activated carbon; zinc cation; metoprolol; clofibric acid; water treatment

\section{Introduction}

Water is necessary to life. It is therefore needed to guarantee a supply of safe water having a good quality, and also to develop efficient treatment of wastewater before its rejection in an 
aqueous medium for environment and biodiversity preservation. Recently, the improvement of analytical technologies has revealed diffusions and increasing pollutions of ground and surface water by numerous organic and mineral compounds present at very low concentrations $[1,2]$. These contaminants, including heavy metals, dyes, personal-care products, plasticizers, reproductive hormones, pesticides and pharmaceuticals residues, are arising from domestic, agricultural and industrial sources.

Adsorption has become a well-established technique to remove pollutants from water. Among various adsorbents used for the waste water treatment processes, activated carbon (AC) powder or granules are efficient to remove organic compounds, especially at low pollutant concentrations, because of their developed nanotexture (high and easily accessible porous surface) and their mostly hydrophobic character [3,4]. Clays are natural and low cost adsorbents used as geochemical barriers, which are able to trap heavy metals and cationic organic species, and therefore to treat efficiently polluted industrial sites and waste landfill leachates [5,6]. Nevertheless, some contaminants, such as polar pesticides and organic derivatives, are still detected at the output of water treatments units, showing limitations of conventional treatment processes [7].

Biopolymers, such as chitosan (CS), are particularly interesting in this domain, not only because they can act as a dispersing phase, but also because they possess inherent interesting features, such as amphiphilicity, tunable topology and porosity at different scales [8,9]. CS is a multifunctional amino-carbohydrate obtained by the partial deacetylation of natural chitin. The presence of amino groups or protonated $\mathrm{NH}_{3}{ }^{+}$groups on the polymer backbone (Figure 1) confers its unique properties: (i) Metal/organic molecules chelating ability [10]; and (ii) absorption of organic compounds through electrostatic interactions [11]. CS can allow different shaping possibilities for hybrid systems, such as hydrogel beads [12].

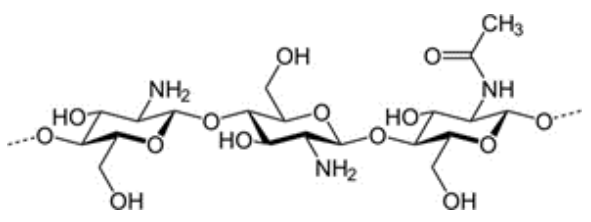

(a)

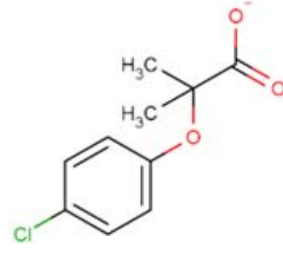

(b)

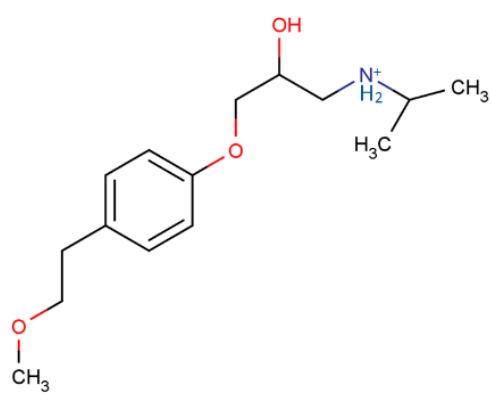

(c)

Figure 1. Molecular structures and speciation of chitosan (CS) (a); clofibric acid (CBA) (b); and metoprolol (MTP) (c) at $\mathrm{pH} 6.5$.

It becomes crucial to develop new hybrid, low-cost and non-toxic adsorbents, presenting a wide adsorption spectrum towards all the pollution types (metals, polar and organic pollutants in variable chemical forms), and therefore are able to operate complete water decontamination at once. By combining chosen adsorbents, such as AC, Clays and CS which possess specific and complementary adsorption properties, a hybrid material adding all the single remediation ability could be tailored, and therefore could handle cationic, anionic and aromatic species at the same time.

Such new hybrid adsorbents having wide adsorption properties could be used either for tertiary water treatment and refining, or for specific treatment of industrial and hospital effluents to get an "at-source" treatment, thus avoiding the mixing of pollutants to reduce difficulty of treatment.

Extensive research performed in our laboratories have demonstrated that AC can efficiently trap organic micropollutants, such as pesticides, and that clay minerals are good candidates for the concentration abatement of toxic metallic ions and cationic molecules in water using cationic exchange $[13,14]$. 
In this work, synthesis, characterization and adsorption performances of a new hybrid adsorbent made of clay minerals and AC powder, which was dispersed in CS biopolymer beads, have been investigated. The adsorption mechanisms have been examined and correlated to the porous texture and the composition of the hybrid material, taking into account the physico-chemical properties of each adsorbent constituting the hybrid material with respect to the adsorbates.

\section{Materials and Methods}

The CS powder $(175,000 \mathrm{~g} / \mathrm{mol})$ was purchased from France CHITIN (Orange, France). SWy-2 montmorillonite of Wyoming (CM) was supplied by the Source Clays Repository of the Clay Minerals Society (Chantilly, VA, USA). The structural formula of the size fraction $(<2 \mu \mathrm{m})$ obtained from the chemical analysis was [10]:

$$
\left(\mathrm{Ca}_{0.13} \mathrm{Na}_{0.34} \mathrm{~K}_{0.03}\right)\left[\mathrm{Al}_{3.04} \mathrm{Fe}(\mathrm{III})_{0.41} \mathrm{Mg}_{0.49} \mathrm{Ti}_{0.01}\right]\left[\mathrm{Si}_{7.98} \mathrm{Al}_{0.02}\right] \mathrm{O}_{20}(\mathrm{OH})_{4}
$$

The basic AC powder prepared from coconut by activation in a steam of water at $900{ }^{\circ} \mathrm{C}$ was supplied by DACARB Company (Asnières sur Seine, France). Zinc chloride, purchased from Aldrich (Saint-Quentin-Fallavier, France), was used as a model for water contamination caused by heavy metals. Two organic molecules, metoprolol (MTP) and clofibric acid (CBA) (Table 1), purchased from Aldrich, were selected as organic pollutant models; CBA was an anti-cholesterol in the anionic form and MTP was a beta blocker in the cationic form at $\mathrm{pH}$ of natural water $(\mathrm{pH}=6.5)$ (Figure 1).

Table 1. Physicochemical characteristics of the adsorbates at $\mathrm{pH} 7$.

\begin{tabular}{cccc}
\hline Pollutant & $\mathrm{p} \boldsymbol{K}_{\mathrm{A} 1} / \mathrm{p} \boldsymbol{K}_{\mathrm{A} 2}$ & $\boldsymbol{V}\left(\AA^{3}\right)$ & $\log \boldsymbol{K}_{\text {ow }}$ \\
\hline CBA & 3.4 & 192 & -0.02 \\
MTP & $9.5 / 14$ & 644 & -0.81 \\
\hline
\end{tabular}

Firstly, the synthesis of pure and hybrid CS beads containing clay mineral and AC powders was investigated. The CS powder was dissolved in acetic acid with a concentration of $0.5 \mathrm{~mol} / \mathrm{L}$ to obtain a former gel mass concentration of $1.75 \%$. The clay minerals and the AC powders (sieving $\leq 50$ microns) with a mass ratio of $3 \%$ were dispersed in the same volume of the acetic acid with the concentration of $0.5 \mathrm{~mol} / \mathrm{L}$ by using an ultrasonic probe at a working frequency of $20 \mathrm{kHz}$ for one hour. Then, in order to reach a homogenous CS concentration in the gel up to 3\%, a small amount of CS was added step by step in the mixture to get the desired concentrations. When all the components were added (CS and charges), the dispersion mixture was stirred for $24 \mathrm{~h}$.

The dispersion mixture was then flowed by using a peristaltic pump at $20 \mathrm{~mL} / \mathrm{min}$ into a $\mathrm{NaOH}$ solution (10\% weight), through a 1.2-mm-diameter syringe. Hybrid CS beads with a diameter of $2 \mathrm{~mm}$ were obtained, having good size homogeneity, required mechanical properties and chemical stability. In this work, five types of beads were studied: Pure CS bead (1-CS), two-component hybrid CS beads containing $1.5 \%$ of montmorillonite (2-CS/CM1.5), 4.5\% of montmorillonite (2-CS/CM4.5) and 1.5\% of AC (2-CS/AC1.5), and three-component hybrid beads containing $1 \%$ of montmorillonite and $0.5 \%$ of AC (3-CS/CM 1/AC 0.5) (Figure 2). 


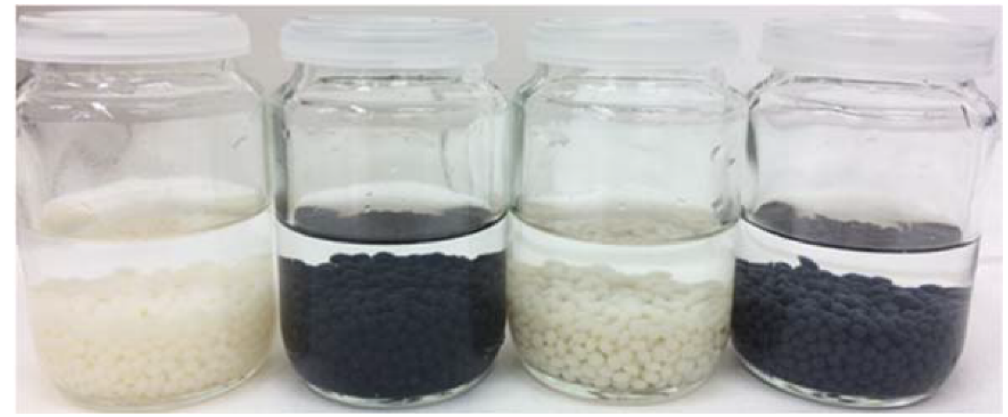

Figure 2. Photograph of pure and hybrid hydrogel CS beads. The mean diameter of the beads was $2 \mathrm{~mm}$.

The porous texture and homogeneity of the hybrid material were characterized by electronic scanning microscopy Cryo-SEM (HITACHI S4500, Tokyo, Japan). After immersion at $-210^{\circ}$, the beads were fractured and introduced in the transfer chamber, and ice was sublimated at $-70{ }^{\circ} \mathrm{C}$ under vacuum $\left(10^{-3} \mathrm{~Pa}\right)$ in order to preserve the porous network free of water to allow its observation.

Nitrogen adsorption-desorption experiments at 77 K (ASAP 2020, Micromeritics, Norcross, GA, USA) were performed to obtain information on the BET specific surface area (Brunauer Emmett Teller method, $P / P_{0}$ ranging from 0.05 to 0.3 ) of the powdered samples and freeze-dried beads after degassing under vacuum $\left(2 \mu \mathrm{m} \mathrm{Hg}, 80^{\circ} \mathrm{C}, 24 \mathrm{~h}\right)$.

The raw materials (each component of the hybrid) and the prepared hydrogel beads were analyzed by thermogravimetric analyses (TGA, Labsys TM SETARAM, Lyon, France) under nitrogen low-flow $(1 \mathrm{~L} / \mathrm{h})$ in order to quantify the amounts of water and each component in the beads, i.e., polymer, clay and AC. The temperature was varied from 20 to $800{ }^{\circ} \mathrm{C}$ with a heating rate of $10{ }^{\circ} \mathrm{C} / \mathrm{min}$. Powdered X-ray diffraction patterns were recorded with a Thermo Electron ARL'XTRA diffractometer (Waltham, MA, USA) equipped with a silicon-lithium solid-state detector, to measure the $d_{001}$ interlayer distance of clay minerals and to evaluate the ability of the biopolymer to intercalate between the montmorillonite layers.

The adsorption properties of $\mathrm{Zn}^{2+}, \mathrm{CBA}$ and MTP have been investigated on the single adsorbents and the hybrid materials. Ultra-pure water $\left(\sigma_{\text {water }}=0.055 \mu \mathrm{S} \cdot \mathrm{cm}^{-1}\right)$ was used as a solvent. The adsorption kinetics of $100 \mathrm{~mL}$ solutions of $\mathrm{Zn}^{2+}, \mathrm{CBA}$ and MTP (concentration $=1.5 \mathrm{mmol} \cdot \mathrm{L}^{-1}$ ) were studied at $25^{\circ} \mathrm{C}$ and $\mathrm{pH} 6.5$ using $200 \mathrm{mg}$ of single or hybrid adsorbents.

The pollutant concentrations were analyzed along with kinetics studies by UV spectroscopy (Cary100, Agilent, Santa Clara, CA, USA) at 224/279 and 229/274 nm for CBA and MTP, respectively. In the case of zinc, atomic absorption spectroscopy (Z-8100, Hitachi, Tokyo, Japan) was used and calibration curves were established in the range of $0.5-1.5 \mathrm{ppm}(\lambda=213.9 \mathrm{~nm}$, the detection limit $=0.008 \mathrm{ppm}$ ).

In order to understand the adsorption mechanisms and determine the key parameters governing adsorption, kinetics studies on single pristine adsorbent (clay or AC or CS) and on hybrid beads were studied. 


\section{Results and Discussion}

\subsection{Characteristics of the Hybrid Material}

\subsubsection{Chemical Composition of the Hybrid Beads}

Thermogravimetric analysis has allowed quantifying the amount of each component in the hybrid $\mathrm{CS} /$ clay beads. In the range of $25-225{ }^{\circ} \mathrm{C}$, the mass loss corresponded to water content. Between 250 and $400{ }^{\circ} \mathrm{C}$, the decomposition of chitosan (CS) occurred and then montmorillonite dehydroxylation took place until $700{ }^{\circ} \mathrm{C}$. The mass losses showed that the 2-CS/CM1.5 beads contained approximately $90 \%$ of water, $8 \%$ of CS and $2 \%$ of montmorillonite, and that the 2 -CS/CM4.5 ones contained $87 \%$ of water, $7 \%$ of CS and $6 \%$ of montmorillonite (Table 2). For the carbon hybrid beads, only the amounts of water and CS was measured to be $90 \%$ and $3 \%$, respectively.

Table 2. Contents of water and chitosan (CS) determined by thermogravimetric analysis.

\begin{tabular}{cccc}
\hline Material & $\begin{array}{c}\text { 1st Mass Loss } \Delta \mathrm{m} / \mathbf{m}(\%) \\
\mathbf{2 5 < T}\left({ }^{\circ} \mathrm{C}\right)<\mathbf{2 2 5}\end{array}$ & $\begin{array}{c}\text { 2nd Mass Loss } \Delta \mathrm{m} / \mathrm{m}(\%) * * \\
\mathbf{2 5 0 < T}\left({ }^{\circ} \mathrm{C}\right)<400\end{array}$ & $\begin{array}{c}\text { 3rd Mass Loss } \Delta \mathrm{m} / \mathrm{m}(\%) \\
\mathbf{4 7 5}<\mathbf{T}\left({ }^{\circ} \mathrm{C}\right)<800\end{array}$ \\
\hline 1-CS & 90 & 3 & - \\
2-CS/AC1.5 & 87 & 4 & - \\
2-CS/CM1.5 & 90 & 8 & 6 \\
2-CS/CM4.5 & 87 & 7 & 0.6 \\
3-CS/CM1/AC0.5 & 86 & 2.2 & 6 \\
\hline * The first mass loss corresponded to the water content; ** the second mass loss corresponded to CS degradation.
\end{tabular}

\subsubsection{Microtexture and Porosity of the Hybrid Beads}

Figure 3 presents the microstructures of three types of beads observed by Cryo-SEM. 1-CS beads had a homogeneous porous structure. Chains of CS (Figure 3a) formed a homogeneously-distributed network. The clay-mineral particles were homogeneously dispersed in the polymer matrix in the two types of hybrid CS beads (Figure 3b). Few large montmorillonite aggregated (around 2-5 $\mathrm{mm}$ diameter), and activated carbons particles were rarely observed (Figure 3c,d). Thus, the hybrid beads appeared homogenous with a high dispersion of the mineral charges within the polymer network, which ensured an intimate charge-polymer mixing.

The presence of the montmorillonite in the beads did not affect the porous network of the hybrid beads. The accessibility of the porous network between the polymer chains allowed the pollutants to be adsorbed on the reactive sites of CS. As far as adsorption properties were concerned, the tailoring of a porous hybrid material was crucial to ensure the pollutant mobility.

The incorporation of AC with a concentration of $1.5 \%$ in the two-component beads has significantly increased the BET specific surface area of the hybrid CS beads (from 35 to $174 \mathrm{~m}^{2} / \mathrm{g}$ ), confirming the important dispersion of AC particles in the polymer matrix (Table 3). However, when the concentration of AC is low, e.g., $0.5 \%$, the porosity remained unchanged, probably due to the presence of montmorillonite and its ability to intercalate CS.

Table 3. BET Specific surface area $\left(\mathrm{S}_{\mathrm{BET}}\right)$ of the raw and hybrid adsorbents.

\begin{tabular}{cc}
\hline Material & $\mathbf{S}_{\text {BET }}\left(\mathbf{m}^{2} / \mathbf{g}\right)$ \\
\hline CS & 2 \\
AC & 811 \\
CM & 35 \\
$1-C S$ & 35 \\
$2-C S / A C 1.5$ & 174 \\
$3-C S / C M 1 /$ AC 0.5 & 35 \\
\hline
\end{tabular}




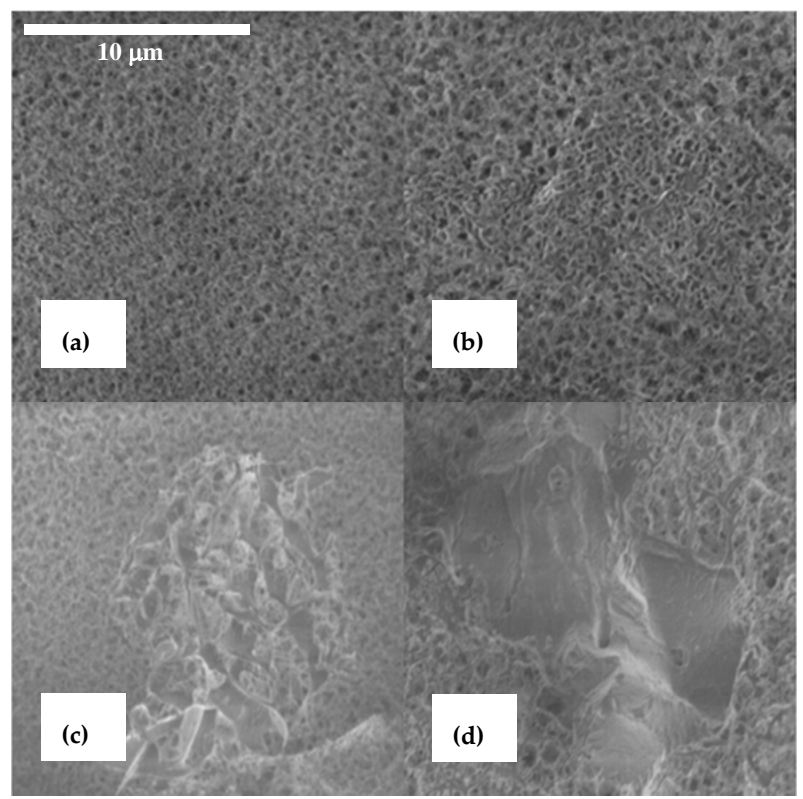

Figure 3. Cryo-SEM images $(\times 5000)$ of 1-CS (a), 2-CS/CM1.5 (b), 2-CS/AC1.5 (c) and 3-CS/CM1/AC0.5 (d).

\subsubsection{Chitosan-Clay Interaction}

X-ray diffraction patterns were performed on the clay sample and on the two hybrid CS-clay beads (Figure 4). The XRD patterns with $2 \theta_{\mathrm{Cu}}$ in the range from $1^{\circ}-16^{\circ}$ show the 001 reflections of the montmorillonite. The basal spacing of the raw montmorillonite sample $\left(2 \theta_{\mathrm{Cu}}=7.6^{\circ}, d_{001}=1.16 \mathrm{~nm}\right)$ was shifted to the small angles after interaction with CS in the hybrid beads $\left(2 \theta_{\mathrm{Cu}}=5.9^{\circ}, d_{001}=1.50 \mathrm{~nm}\right)$. The intercalation of CS in the interlayer is then clearly shown. The increase in the basal spacing value $\left(d_{001}=0.34 \mathrm{~nm}\right)$ for the two clay hybrid beads (2-CS/CM1.5 and 2-CS/CM4.5) indicates that the CS molecules were in a monolayer arrangement $[15,16]$. This is in agreement with the $d_{001}$ value of $0.38 \mathrm{~nm}$, representing the thickness of one CS layer, measured from the XRD pattern of a CS film [17]. Due to the partial cationic nature of CS at pH 6.5 (Figure 4), the intercalation of CS monolayers in the montmorillonite clay may take place through a cationic exchange procedure between $-\mathrm{NH}_{3}{ }^{+} \mathrm{CS}$ chains groups and the positively charged interlayer sites of the clay minerals previously occupied by the exchangeable cations.

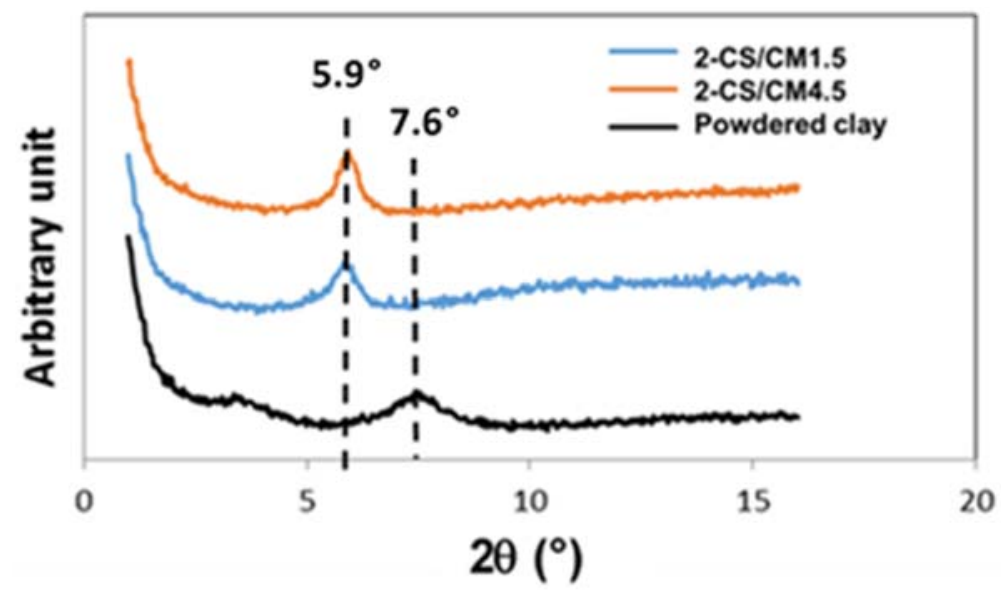

Figure 4. X-ray diffraction patterns of the clay sample and the clay containing hybrid beads. 


\subsection{Adsorption Studies}

Adsorption results on raw and hybrid materials are summarized in Table 4. First-order [18] or second-order [19] models were used to calculate $Q_{\mathrm{t}}$ values, which were shown as follows, respectively:

$$
\begin{aligned}
& Q_{\mathrm{t}}=Q_{\mathrm{e}} \cdot\left(1-\mathrm{e}^{-k t}\right) \\
& Q_{\mathrm{t}}=\frac{k_{2} \cdot Q_{\mathrm{e}}^{2} \cdot t}{1+\left(k_{2} \cdot \mathrm{Q}_{\mathrm{e}} \cdot t\right)}
\end{aligned}
$$

$Q_{\mathrm{t}}$ is the adsorbed amount as a function of time $(t) ; Q_{\mathrm{e}}$ is the maximum adsorbed amount $(\mathrm{mmol} / \mathrm{g})$; $k$ is the velocity constant in $\mathrm{min}^{-1}$; and $k_{2}$ is the velocity constant $\left(\mathrm{g} \cdot \mathrm{mmol}^{-1} \cdot \mathrm{min}^{-1}\right)$.

Adsorption kinetic experiments have been carried out and the adsorbed amounts were determined and compared in the same experimental conditions.

In order to examine the adsorption mechanisms and quantify the role of each adsorbent in the hybrid material, the charge and the speciation of the adsorbent and the species of the adsorbates are presented in Figure 5. Under working conditions, AC was positively charged, clay minerals were negatively charged and for CS, half of the amino groups were protonated.

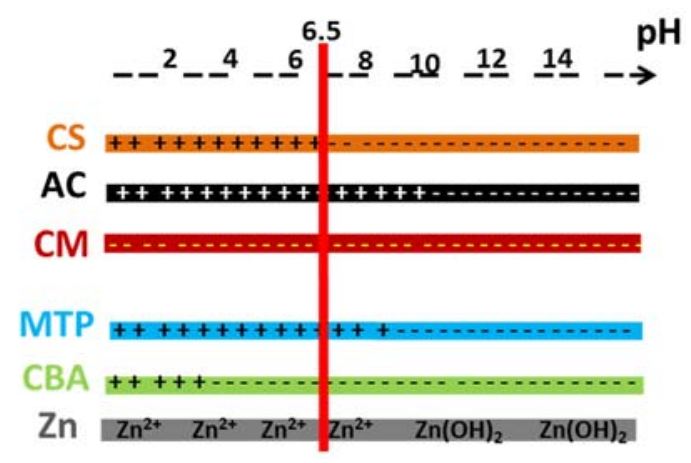

Figure 5. Charges of adsorbents and pollutants as a function of $\mathrm{pH}$. The red line corresponds to the working $\mathrm{pH}$ value.

Table 4. Maximum adsorbed amounts $Q_{\mathrm{e}}$ calculated from adsorption kinetics of $\mathrm{Zn}^{2+}$, Metoprolol (MTP) and clofibric acid (CBA) using the second-order model, or the first-order model (*).

\begin{tabular}{cccc}
\hline Material & \multicolumn{3}{c}{$Q_{\mathbf{e}}(\mathbf{m m o l} / \mathbf{g})$ or $\mathbf{( m g / g )}$} \\
\hline Raw adsorbent & $\mathbf{Z n}^{2+}$ & MTP & CBA \\
\hline \multirow{2}{*}{ CS } & 0.14 & 0.02 & 0.05 \\
& 9 & 2 & 12 \\
AC & 0.09 & 0.60 & 0.56 \\
& 6 & 161 & 120 \\
CM & 0.24 & 0.48 & 0.02 \\
& 15 & 129 & 5 \\
\hline Hybrid adsorbent & $\mathbf{Z n}^{2+}$ & MTP & CBA \\
\hline \multirow{2}{*}{ 1-CS } & 0.14 & 0.01 & 0.04 \\
& 9 & $* 1$ & 9 \\
2-CS/AC1.5 & 0.16 & $0.10 *$ & 0.10 \\
& 10 & 26 & 21 \\
2-CS/CM1.5 & 0.10 & 0.11 & 0.04 \\
& 6 & 30 & 8 \\
3-CS/CM1/AC0.5 & 0.16 & 0.07 & 0.07 \\
& 10 & 19 & 16 \\
\hline
\end{tabular}




\subsubsection{Adsorption of $\mathrm{Zn}^{2+}$}

Interesting adsorbed amounts of zinc cations were measured on raw CS and clay-mineral powders, ranging from 6 to $15 \mathrm{mg} / \mathrm{g}$ (Table 4). Adsorption kinetics of $\mathrm{Zn}^{2+}$ is presented in Figure 6. As expected, montmorillonite is the best adsorbent for $\mathrm{Zn}^{2+}$, as far as cationic exchange occurs and chemisorption with deprotonated silanols (and aluminols) as well (Figure 5). CS also presents interesting adsorption capacities because it contains few deprotonated hydroxyl groups and is able to give electrostatic interactions with zinc, which mainly include complexion with non-binding doublets on nitrogen and oxygen atoms (Figure 1a). For the beads, same adsorption properties were observed. The adsorption properties of the CS beads containing AC was slightly increased, compared to the pure CS beads, demonstrating that the active sites of CS might be more accessible to zinc adsorption. The AC addition increased the BET surface area which became five times higher than those without AC. On the contrary, the incorporation of montmorillonite with a concentration of $1.5 \%$ in the CS beads shows that the two intercalation of the montmorillonite by the CS chains has decreased: (i) The adsorption activity of CS possibly by positively charging the amine groups in the interlamellar space $\left(\mathrm{NH}_{3}{ }^{+}\right)$, and (ii) the adsorption activity of clay minerals by removing the exchangeable cations from the interlayer space. An additional experiment showed that, an increasing rate of the montmorillonite content with a concentrate range from $1.5 \%$ to $4.5 \%$ in the CS-clay-mineral composite was responsible for a significant improvement of the adsorbed amounts of zinc cations (33\%), though this uptake was still lower that the one of raw montmorillonite (kinetic not shown in Figure 6). These results showed that despite the intercalation of a part of CS chains in the clay minerals, the porous network of the beads ensured a good transport of the solution to the remaining reactive sites of the montmorillonite.

The adsorption ability towards zinc cation on the three-component beads was stronger than on the CS-montmorillonite composites, owing to the addition of AC.

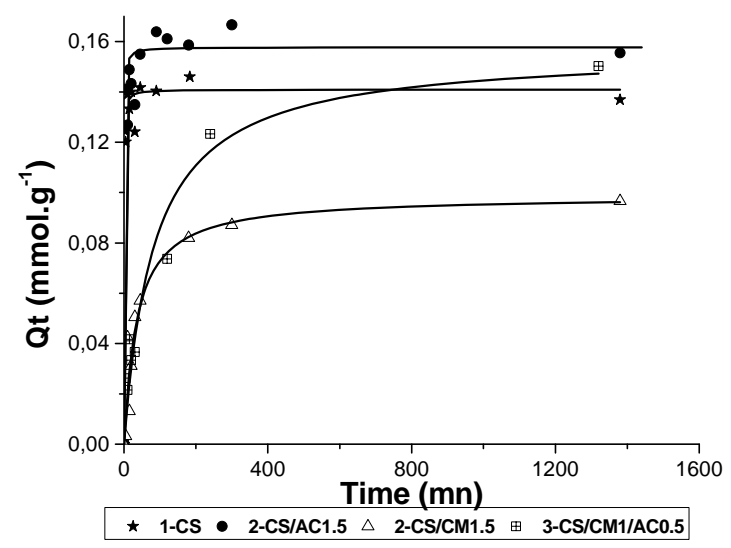

Figure 6. $\mathrm{Zn}^{2+}$ adsorption kinetics on pure and hybrid hydrogel CS beads. The continuous lines represent the calculated data using the first-order model.

\subsubsection{Adsorption of MTP}

Adsorption kinetics of MTP was presented in Figure 7. As expected, CS (raw and CS beads) was a weak adsorbent for MTP (Table 4) because of the electrostatic repulsions between CS chains and MTP.

Through the same mechanism observed previously for zinc, MTP was well adsorbed $(129 \mathrm{mg} / \mathrm{g}$, $0.48 \mathrm{mmol} / \mathrm{g}$ ), by raw clay minerals having a cationic exchange of $1 \mathrm{mmol} / \mathrm{g}$. MTP could interact with the montmorillonite silanols sites, but it possessed a planar molecular structure and was also able to intercalate easily into the clay structure and adsorb through cationic exchange. The incorporation of montmorillonite in the hybrid beads allowed the material to adsorb few MTP organic cation (uptake of $30 \mathrm{mg} / \mathrm{g})$. 
Pure AC showed a highly-adsorbed amount of $161 \mathrm{mg} / \mathrm{g}$ for MTP. MTP was assumed to be adsorbed through Van der Waals $\pi \rightarrow \pi$ interactions, despite electrostatic repulsions. The incorporation of $1.5 \%$ of $\mathrm{AC}$ in the CS beads improved the adsorbed amount of the beads to $26 \mathrm{mg} / \mathrm{g}$. As a result, the adsorption abilities of the three-component beads were twenty-five times higher than for pure CS beads showing the benefits of montmorillonite and AC charge addition. Synergic effects occurred, coming from an increase of the hybrid beads porosity and a good accessibility of adsorption sites of each adsorbent.

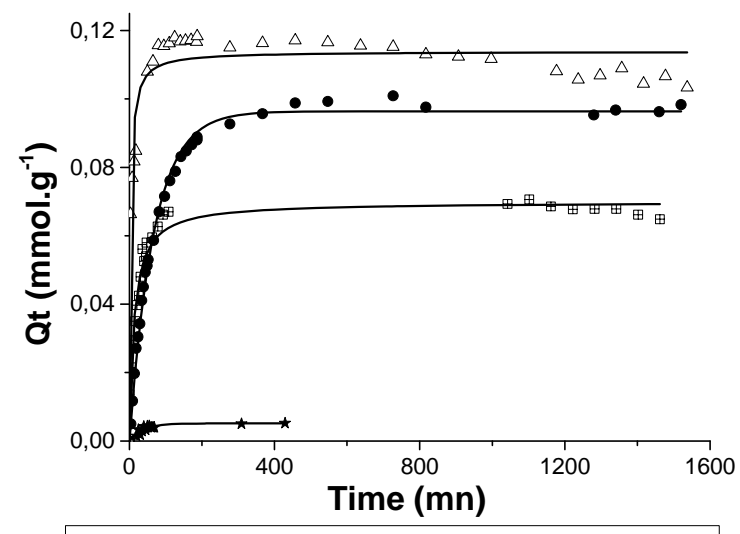

$\star$ 1-CS $\bullet 2-C S / A C 1.5 \triangle 2-C S / C M 1.5 \boxplus$ 3-CS/CM1/AC0.5

Figure 7. MTP adsorption kinetics of pure and hybrid hydrogel CS beads. The continuous lines represent the calculated data using the best-fitting model.

\subsubsection{Adsorption of CBA}

The adsorption kinetics of CBA is displayed in Figure 8. Raw CS and pure CS beads (1-CS) showed an adsorbed amount of $12 \mathrm{mg} / \mathrm{g}$ and $9 \mathrm{mg} / \mathrm{g}$ (Table 4) towards CBA anionic molecules, respectively. At $\mathrm{pH}$ 6.5, half of the amine groups (in the range from 2.5 to $5 \mathrm{mmol} / \mathrm{g}$ theoretical value) of CS, having a $\mathrm{pK} \mathrm{A}_{\mathrm{A}}$ of 6.3 , were protonated (Figure 5). These results demonstrate that, due to its $-\mathrm{NH}_{3}{ }^{+}$sites giving electrostatic interactions with anions, CS is therefore a good candidate to adsorb negatively charged organic molecules, such as CBA.

Pure AC showed a high adsorption affinity with CBA through mainly $\pi \rightarrow \pi$ interactions together with minor Van der Waals interactions ones, giving the highest adsorbed amount of $120 \mathrm{mg} / \mathrm{g}$ (Table 4). As it was shown previously for MTP adsorption, the incorporation of AC with a concentration of $1.5 \%$ in the CS beads significantly improved the adsorbed amount of the beads towards CBA (from 9 to 21 $\mathrm{mg} / \mathrm{g})$.

As expected, montmorillonite was the worst adsorbent for CBA anions, due to its negative surface charge (Figure 5). Thus, the incorporation of this clay mineral with a concentration of $1.5 \%$ in the CS beads slightly decreased the adsorbed amount of the beads because the intercalation of CS chains between the clay layers hindered some CS adsorption sites.

In the three-component beads, the introduction of AC with a concentration of $0.5 \%$ led to an enhancement of the adsorbed amount, reaching $21 \mathrm{mg} / \mathrm{g}$, which was twice the ones of raw CS $(9 \mathrm{mg} / \mathrm{g}$ for CS) or CS-clay mineral hybrid (8 mg/g for 2-CS/CM1.5) beads. 


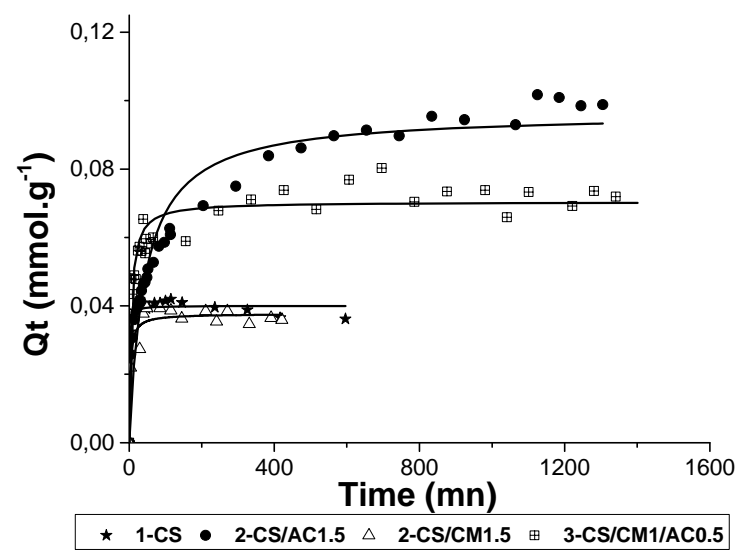

Figure 8. Adsorption kinetic of CBA of pure and hybrid hydrogel CS beads. The continuous lines represent the calculated data using the first-order model.

\section{Conclusions}

A new hybrid porous material was successfully prepared. The obtained hybrid CS beads containing montmorillonite and activated carbon possessed the required mechanical properties and are homogeneous. The adsorption performances of the hybrid beads verified the accessibility and connection of the porous network. The beads showed also the advantage of easy handling and recovery in a water purification process.

The main interactions between CS beads and $\mathrm{Zn}^{2+}$ were chelating interactions with nitrogen- and oxygen-electron-lone pairs. Pure CS bead affinity is higher for $\mathrm{Zn}^{2+}$ than for MTP because CS contains only few deprotonated-hydroxyl groups at $6.5 \mathrm{pH}$, was able to give electrostatic interactions with MTP. CS beads showed a moderate adsorption ability for anionic CBA, in comparison to cationic MTP. As it was expected, CS was also very useful to adsorb negatively- charged organic molecules due to its $-\mathrm{NH}_{3}{ }^{+}$sites through electrostatic interactions. Adsorption properties of CS towards anionic species could be enhanced at lower $\mathrm{pH}$. Despite the intercalation of the CS chains into the interlayer spacing, altering the hybrid beads porosity, the CS-montmorillonite beads were efficient towards metallic $\left(\mathrm{Zn}^{2+}\right)$ and organic (MTP) cations. Synergic effects were obtained for MTP, which is trapped by the montmorillonite aluminols and silanols groups.

Although the raw AC materials are known to be more efficient for the adsorption of hydrophobic organic pollutants, the hybrid CS/carbon beads showed a wider spectrum of adsorption and synergic effects, especially towards $\mathrm{Zn}^{2+}$. The incorporation of $\mathrm{AC}$ has improved the porosity of the hybrid material and is effective for anionic and cationic organic pollutants, due to hydrophobic interactions and more generally Van der Waals interactions. Moderate adsorbed amounts were obviously measured as compared to raw-material adsorption properties. Synergic effects were obtained in the hybrid materials for CBA and MTP adsorption. The complementary adsorption properties of clay, AC and CS were preserved in the hybrid three-component material, leading to an adsorbent having large adsorption properties for different kinds of water contaminants, i.e., metals, negativelyor positively-charged organic molecules. It could be also used to purify tap water contaminated with pesticides, nitrates, and pharmaceutical derivatives. The re-generation of the loaded beads is under progress and promising behaviors are expected.

Author Contributions: M.C., F.B., S.D.-O. and F.M. conceived, prepared and characterized the hybrid materials, made the adsorption study and wrote the article; L.D. and L.R. performed thermal and gas adsorption analyses; R.K.D. brought advises for the choice of (CS) type and for discussions; M.L. and H.B.Y. brought advices for the protocol to prepare the hydrogels and for discussions.

Funding: This research was funded by the Centre Val de Loire region, France (MatHéO project).

Conflicts of Interest: The authors declare no conflicts of interest. 


\section{References}

1. Huerta-Fontela, M.; Galceran, M.T.; Martin-Alonsoa, J.; Ventura, F. Occurrence of psychoactive stimulatory drugs in waste waters in north-eastern Spain. Sci. Total Environ. 2008, 397, 31-40. [CrossRef] [PubMed]

2. Stavrakakis, C.; Colin, R.; Faur, C.; Hequet, V.; Le Cloirec, P. Analysis and behavior of endocrine disrupting compounds in wastewater treatment plant. Eur. J. Water Qual. 2008, 39, 145-156. [CrossRef]

3. De Ridder, D.J.; Verliefde, A.R.D.; Schoutteten, K.; Van der Linden, B.; Heijman, S.G.J.; Beurroies, I.; Denoyel, R.; Amy, G.L.; Van Dijk, J.C. Relation between interfacial energy and adsorption of organic micropollutants onto activated carbon. Carbon 2013, 53, 153-160. [CrossRef]

4. Ocampo-Pérez, R.; Abdel daiem, M.M.; Rivera-Utrilla, J.; Méndez-Díaz, J.D.; Sánchez-Polo, M. Modeling adsorption rate of organic micropollutants present in landfill leachates onto granular activated carbon. J. Colloid Interface Sci. 2012, 385, 174-182. [CrossRef] [PubMed]

5. Gautier, M.; Muller, F.; Beny, J.M.; Le Forestier, L.; Alberic, P.; Baillif, P. Interactions of ammonium smectite with low-molecular-weight carboxylic acids. Clay Miner. 2009, 44, 207-219. [CrossRef]

6. Bright, M.I.; Thornton, S.F.; Lerner, D.N.; Tellam, J.H.H. Attenuation of landfill leachate by clay liner materials in laboratory columns, Experimental procedures and behavior of organic contaminants. Waste Manag. Res. 2000, 18, 198-214. [CrossRef]

7. Delpeux-Ouldriane, S.; Gineys, M.; Masson, S.; Cohaut, N.; Reinert, L.; Duclaux, L.; Béguin, F. Adsorption and desorption of emerging water contaminants on activated carbon fabrics. Int. J. Environ. Pollut. Remediat. 2016, 4, 1-6. [CrossRef]

8. Crini, G.; Badot, P.M.; Grubal, E. Chitin et Chitosane du Biopolymère à L'application; Presses Universitaires de Franche-Comté: Besançon, France, 2009; pp. 10-30.

9. Crini, G.; Badot, P.M.; Morin Crini, N. Traitement des eaux par du chitosane: Interêt, méthodes et perspectives. Techniques de L'ingénieur 2009, 126, 1-13.

10. Lei, Z.; Yuexian, Z.; Zhengjun, C. Removal of heavy metal ions using chitosan and modified chitosan: A review. J. Mol. Liq. 2019, 214, 175-191.

11. Soni, U.; Bajpai, J.; Singh, S.K.; Bajpai, A.K. Evaluation of chitosan-carbon based biocomposite for efficient removal of phenols from aqueous solutions. J. Water Process Eng. 2017, 16, 56-63. [CrossRef]

12. Hydari, S.; Sharififard, H.; Nabavinia, M.; Parvizi, M.R. A comparative investigation on removal performances of commercial activated carbon, chitosan biosorbent and chitosan/activated carbon composite for cadmium. Chem. Eng. J. 2012, 193-194, 276-282. [CrossRef]

13. Delpeux-Ouldriane, S.; Gineys, M.; Cohaut, N.; Béguin, F. The role played by local pH and pore size distribution in the electrochemical regeneration of carbon fabrics loaded with bentazon. Carbon 2015, 94, 816-825. [CrossRef]

14. Ghayaza, M.; Le Forestier, L.; Muller, F.; Tournassat, C.; Beny, J.M. Pb(II) and Zn(II) adsorption onto Na- and Ca-montmorillonites in acetic acid/acetate medium: Experimental approach and geochemical modeling. J. Colloid Interface Sci. 2011, 361, 238-246. [CrossRef] [PubMed]

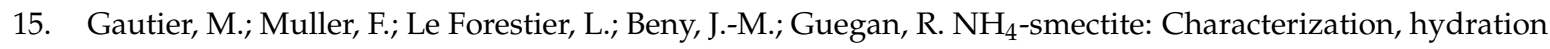
properties and hydro mechanical behavior. Appl. Clay Sci. 2010, 49, 247-254. [CrossRef]

16. Wang, S.F.; Shen, L.; Tong, Y.J.; Chen, L.; Phang, I.Y.; Lim, P.Q.; Liu, T.X. Biopolymer chitosan/montmorillonite nanocomposites: Preparation and characterization. Polym. Degrad. Stab. 2005, 90, 123-131. [CrossRef]

17. Darder, M.; Colilla, M.; Ruiz-Hitzky, E. Chitosan-clay nanocomposites: Application as electrochemical sensors. Appl. Clay Sci. 2005, 28, 199-208. [CrossRef]

18. Lagergren, S.K. About the Theory of So-called Adsorption of Soluble Substances. Hanlingar 1898, 24, 1-39.

19. Ho, Y.S.; McKay, G. Pseudo-second order model for sorption processes. Process. Biochem. 1999, 34, 451-465. [CrossRef]

(C) 2018 by the authors. Licensee MDPI, Basel, Switzerland. This article is an open access article distributed under the terms and conditions of the Creative Commons Attribution (CC BY) license (http:/ / creativecommons.org/licenses/by/4.0/). 